PAwEŁ SzEPTyCKI ${ }^{\dagger}$ (Lawrence, KS)

\title{
INTEGRATION OF POLYNOMIALS
}

Abstract. We prove that the only functions for which certain standard numerical integration formulas are exact are polynomials.

For a set $(\tau)=\left(0 \leq \tau_{0}<\tau_{1}<\ldots<\tau_{N} \leq 1\right)$ we consider the numerical integration formula

$$
\int_{a}^{b} f(t) d t \approx(b-a) \sum_{l=0}^{N} \alpha_{l} f\left(a+\tau_{l}(b-a)\right) .
$$

If we require that the formula be exact, i.e., the $\approx$ sign can be replaced by the $=$ sign, for all polynomials of degree at most $m$ and for all intervals $[a, b]$, then letting $a=0, b=x$ and $f(t)=t^{l}$, we get

$$
\sum_{k=0}^{N} \alpha_{k} \tau_{k}^{l}=\frac{1}{l+1}, \quad l=0, \ldots, m .
$$

For $m=N$ this is an $(N+1) \times(N+1)$ system of linear equations and $(\alpha)=\left(\alpha_{k}\right)$ are uniquely determined in the case when $m=N$. It may but need not happen that (2) remains valid for integers $l>N$; we denote by $m_{(\tau)}$ the largest integer with this property, i.e., (1) is exact for all polynomials of degrees at most $m_{(\tau)}$ but not for polynomials of higher degree. The integer $m_{(\tau)}$ is referred to as the degree of precision of (1). It is known, e.g., by counting degrees of freedom, that $m_{(\tau)}<2 N+2$ and that $m_{(\tau)}=2 N+1$ is attained if $(\tau)$ are roots of the Legendre polynomial on the interval $[0,1]$. This choice of $(\tau)$ gives rise to the Gaussian rule.

Various choices of the set $(\tau)$ and the corresponding coefficients $(\alpha)$ can be found in texts on numerical analysis, e.g., [2]. Here are two of them.

2000 Mathematics Subject Classification: Primary 65D32.

Key words and phrases: quadrature formulas.

$\dagger$ Professor Paweł Szeptycki died on January 30th, 2004 (Editors). 
For $\tau_{k}=k / N, k=0, \ldots, N$, we get the well known Newton-Cotes formula. In this case $m_{(\tau)}=m_{N}=N$ or $N+1$ depending on whether $N$ is odd or even. We note in passing that for $N=8$ the coefficients $(\alpha)$ change sign giving rise to (numerical) instability of the corresponding integration formula.

Note that the coefficients $(\alpha)$ can also be written explicitly in the form of integrals of Lagrange polynomials for the points $(\tau)$.

Our aim in this note is to prove the following theorem.

Theorem. If $f: \mathbb{R} \rightarrow \mathbb{R}$ is continuous and for some $(\tau)$ satisfies

$$
\int_{a}^{b} f(t) d t=(b-a) \sum_{l=0}^{N} \alpha_{l} f\left(a+\tau_{l}(b-a)\right)
$$

for all intervals $[a, b]$, then $f$ is a polynomial of degree at most $m_{(\tau)}$.

The corresponding result in the case of some of the examples mentioned above was addressed in [1], but already in the case of the Newton-Cotes formula for $N=3$ it was obtained under differentiability assumptions on $f$ which, as it turns out, are unnecessary, even though useful.

We now give the proof of the theorem. First, as in the preceding work quoted above, we assume that $f$ is sufficiently, at least $m_{(\tau)}+1$ times, continuously differentiable. We will show that the derivative $f^{\left(m_{(\tau)}+1\right)}$ is zero. Since the assumption on $f$ is translation invariant, i.e., together with $f$ it is satisfied by every function $f_{x}(t)=f(t+x)$, it is sufficient to show that $f^{\left(m_{(\tau)}+1\right)}(0)=0$. Subtracting from $f$ a polynomial of degree $m_{(\tau)}$ changes neither the hypotheses on $f$ nor the desired conclusion; taking this polynomial to be the Taylor polynomial of $f$ at zero, we may assume that $f^{\left(m_{(\tau)}\right)}(0)=0$. With $a=0, b=x$, from (3) we get

$$
\int_{a}^{x} f(t) d t=x \sum_{k=0}^{N} \alpha_{k} f\left(\tau_{k} x\right) .
$$

Differentiating $m_{(\tau)}+1$ times we get

$$
\begin{aligned}
f^{\left(m_{(\tau)}\right)}(x)= & x \sum_{k=0}^{N} \alpha_{k} \tau_{k}^{m_{(\tau)}+1} f^{\left(m_{(\tau)}+1\right)}\left(\tau_{k} x\right) \\
& +\left(m_{(\tau)}+1\right) \sum_{k=0}^{N} \alpha_{k} \tau_{k}^{m(\tau)} f^{\left(m_{(\tau)}\right)}\left(\tau_{k} x\right) .
\end{aligned}
$$

We now divide the last equality by $x$ and let $x \rightarrow 0$ to conclude that

$$
f^{\left(m_{(\tau)}+1\right)}(0)\left(1-\left(m_{\tau}+2\right) \sum_{k=0}^{N} \alpha_{k} \tau_{k}^{m_{(\tau)}+1}\right)=0 .
$$

This yields the conclusion, since (2) does not hold for $l=m_{(\tau)}+1$. 
We proceed now to the proof in the general case when the function $f$ is merely continuous. The crucial observation here is that the (vector) space $V_{\text {pol }}$ of all polynomials of degree at most $m_{(\tau)}$ is complete in any vector topology. We can for instance consider the following concepts of convergence:

- Convergence of all $\left(m_{(\tau)}+1\right)$ coefficients of a sequence of polynomials in $V_{\mathrm{pol}}$.

- Uniform convergence on every (finite) interval (almost uniform convergence).

- Convergence in each of the norms $\|f\|_{p,-T, T}=\left(\int_{-T}^{T}|f(t)|^{p} d t\right)^{1 / p}$, $1 \leq p<\infty$.

The limit of a sequence in $V_{\text {pol }}$ convergent in any of the modes indicated above is again in $V_{\text {pol }}$.

Consider now the set $V=V_{(\tau)}$ of all continuous functions $f$ satisfying (3) for all intervals $[a, b]$, where the coefficients $(\alpha)$ are determined from (2) with $m=N$.

$V$ is a vector space containing $V_{\text {pol }}$ as a subspace. Moreover, $V$ is closed with respect to almost uniform convergence (i.e., uniform convergence on all finite intervals) and is translation invariant in the sense explained above. These two properties imply that if $\varphi$ is a continuous function vanishing outside an interval, $[-1,1]$ say, then the convolution

$$
\varphi * f(t)=\int \varphi(s) f(t-s) d s
$$

belongs to $V$ whenever $f$ does. Also, if $\varphi$ is differentiable, then $\varphi * f$ is as many times differentiable as $\varphi$ is. It follows then from the first part of the proof that for sufficiently differentiable $\varphi$, and for every $f \in V, \varphi * f \in V_{\mathrm{pol}}$. We choose now a smooth $\varphi$ so that $\varphi=0$ outside $[-1,1], \int \varphi d t=1$ and let $\varphi_{n}(t)=n \varphi(n t)$. Then it is well known and easy to prove that for every continuous $f$ the sequence $f_{n}=\varphi_{n} * f$ converges to $f$ almost uniformly. Hence, every $f \in V$ is a limit of a sequence $\left(f_{n}\right) \subset V_{\text {pol }}$ and $V \subset V_{\text {pol }}$. It follows that $V=V_{\text {pol }}$ and the proof is complete.

A question may be asked as to the minimal natural hypotheses for validity of the theorem. The left hand side of (3) makes sense for $f$ Lebesgue integrable on any finite interval but the right hand side may not be defined if $f$ is not defined everywhere. However, both sides of (3) make sense if $f$ is Riemann integrable on every finite interval. The theorem remains valid also in this case and the proof remains the same except that instead of the almost uniform convergence of the sequence $\left(f_{n}\right)$ above, we use its convergence in the sense of the family of the norms \|\|$_{p}$ for some fixed $p$, e.g., $p=1$.

The author would like to thank Ralph Byers and Weizhang Huang for their useful comments. 


\section{References}

[1] Á. Benyi, P. Szeptycki and F. S. Van Vleck, Archimedean properties of parabolas, Amer. Math. Monthly 107 (2000), 945-949.

[2] S. D. Conte and C. de Boor, Elementary Numerical Analysis, McGraw-Hill, 1980.

Department of Mathematics

University of Kansas

Lawrence, KS 66045, U.S.A.

Received on 15.10.2003 